Classification

Physics Abstracts

$82.70 \mathrm{G}-61.10-61.16 \mathrm{H}$

\title{
GROWTH PROCESS AND STRUCTURE OF ALUMINOSHICATE GELS
}

\author{
J.C. Pouxviel ${ }^{a}$, J.P. Boilot, A. Lecomte ${ }^{+}$and A. Dauger ${ }^{+}$ \\ Groupe de Chimie du Solide, Laboratoire de Physique de la Matière Condensée, Ecole Polytechnique, \\ 91128 Palaiseau Cedex, France \\ +Ecole Nationale Supérieure de Céramique Industrielle, 87065 Limoges Cedex, France
}

(Reçu le 7 janvier 1987, révisé le 26 février 1987, accepté le \& mars 1987)

Résumé.-La croissance et la structure des sols et gels dans le système ester silicique $/ \mathrm{H}_{2} \mathrm{O} /{ }^{\mathrm{i}} \mathrm{PrOH}$ ont été étudiés par RMN et diffusion centrale des rayons X. La structure des particules élémentaires est gouvernée par un processus chimique avec formation de liaisons Al-O-Al et la structure fractale du gel (dimension 1,8) résulte d'une agrégation de clusters.

Abstract.-Small-angle X-ray scattering and $\left({ }^{29} \mathrm{Si}-{ }^{27} \mathrm{Al}\right) \mathrm{NMR}$ were used to study the growth process and structure of sols and gels in the silicon-aluminium ester $/ \cdot \mathrm{H}_{2} \mathrm{O} / \mathrm{i} \mathrm{PrOH}$ system. The structure of elementary particles is governed by a chemical growth process with the formation of Al-O-Al linkages, and the self-similar structure of the gel (fractal dimension of 1.8 ) results from a cluster-cluster growth process.

\section{Introduction}

In a previous paper [1], we reported the preparation of chemically ultrahomogeneous gels in the $\mathrm{SiO}_{2}-\mathrm{Al}_{2} \mathrm{O}_{3}$ system, using new metal-organic precursors with Al-O$\mathrm{Si}$ linkages. We have carried on our investigation of the hydrolysis and gelation of the silicon-aluminium ester in alcoholic solutions using the techniques of ${ }^{29} \mathrm{Si}$ and ${ }^{27} \mathrm{Al} \cdot \mathrm{NMR}$ and small-angle X-ray scattering (SAXS).

Firstly, the growth process has been studied by NMR. It is now well known that the first steps of the gelation can be followed in solution by the appearance and disappearance of signals arising from different low molecular weight oligomers [2-5].

Secondly, SAXS has been used to determine the size and growth kinetics of the polymeric species formed during the crossover from liquid to gel (by analysis of the low angle region) and to obtain information about the structure of these species (by analysis of the intermediate angle Porod region). For polymer-like struc-

a Also at Saint-Gobain Recherche, 39, Quai Lucien Lefranc, 93304 Aubervilliers, France tures [6] (mass-fractal objects) the classic power-law exponent observed in the Porod regime is the fractal dimension $D$ which relates the size $R$ of the objects to its mass $M \sim R^{D}$.

This paper reports on the results of these investigations and shows that, under our experimental conditions, the structure of the inorganic polymer is governed both by a monomer growth process with the formation of elementary clusters and by a cluster-cluster growth process.

\section{Experimental}

The initial mixture consists of the $(\mathrm{EtO})_{3} \mathrm{Si}-\mathrm{O}-\mathrm{Al}$ $(\mathrm{OBu})_{2}$ silicon-aluminium ester, $\mathrm{H}_{2} \mathrm{O}(\mathrm{pH}=2.5-\mathrm{HCl}$ as catalyst) and ${ }^{\mathrm{i}} \mathrm{PrOH}$ in the molar ratio 1-10-4. The formation of a precipitate is observed during the addition of water to the organic solution (silicic ester in 2-propanol). Then, a clear solution is obtained after an instantaneous dissolving of the precipitate in the liquid. The ageing of the solution was carried out at room temperature, in a closed flask; under these conditions, after 15 hours, the mixture turns into a ${ }^{29} \mathrm{Si}$ and ${ }^{27} \mathrm{Al}$ Nuclear magnetic resonance spectra were obtained at 80 and $104 \mathrm{MHz}$ respectively on a 
AM400 wide bore spectrometer. The X-ray scattering data were recorded with a point-like small-angle camera ( $\mathrm{CuK} \alpha$-quartz monochromator). A sample cell is used with "Mylar" windows. The scattered intensity was counted with a position sensitive proportional counter.

\section{NMR results}

Information on the growth process can be obtained form the ${ }^{29} \mathrm{Si}$ NMR spectra because the chemical shifts are very sensitive to the local environment of $\mathrm{Si}$ atoms (for instance the number of $\mathrm{OH}, \mathrm{OR}$, OSi neighbouring groups). After adding the aqueous solution to the organic one and dissolving of the precipitated particles (hereafter referred to as the initial time $: t=0$ ) the ${ }^{29} \mathrm{Si}$ NMR spectrum shows the presence of two predominant Si resonances which do not change by using the proton decoupling technique. These resonances can be assigned to the $(=\mathrm{AlO}) \mathrm{Si}(\mathrm{OR})_{3}$ group $(-84.6$ ppm with respect to TMS peak) and to the (=AlO) $\mathrm{Si}-\mathrm{O}-\mathrm{Si}(\mathrm{OAl}=)$ group $(-91.5 \mathrm{ppm})$ respectively. Consequently, the ${ }^{29} \mathrm{Si}$ NMR spectrum clearly indicates a partial condensation of the starting silicic ester (presence of dimers with Si-O-Si binding).

Just before $t_{g}$, the ${ }^{29} \mathrm{Si}$ NMR spectra are only slightly modified. We can note peaks corresponding to groups of the starting organic precursor and new ones resulting from the substitution of one alkoxy group by one hydroxyl group. However, we do not observe peaks corresponding to other condensed species, indicating that the formation of oligomers with $\mathrm{Si}-\mathrm{O}-\mathrm{Si}$ bonding is weak and that the degree of condensation of $\mathrm{Si}$ atoms has only slightly increased during gelation (from $t=0$ to $t=t_{g}$ ).

Concerning the ${ }^{27} \mathrm{Al}$ spectrum of the pure silicic ester (Fig.1a), an intense sharp line $(\Delta \nu=270 \mathrm{~Hz})$ at $7 \mathrm{ppm}$ (with respect to $\mathrm{Al}\left(\mathrm{H}_{2} \mathrm{O}\right) 6^{3+}$ ) can be confidently assigned to octahedral aluminium species and a broad line $(\Delta \nu=7000 \mathrm{~Hz})$ at $48 \mathrm{ppm}$ to tetrahedrally coordinated aluminium atoms [7].

After the addition of water $(t=0)$, the sharp line disappears on the ${ }^{27} \mathrm{Al}$ NMR spectra (Fig.1b) while another one is formed at $49.4 \mathrm{ppm}$. This relatively narrow $\mathrm{Al}$ resonance $(\Delta \nu=150 \mathrm{~Hz})$ presumably arises from symmetric tetrahedral aluminium sites, resulting from a decrease of the molecular complexity. In fact, besides the sharp line due to 4-coordinated $\mathrm{Al}$ atoms which only represents $20 \%$ of $\mathrm{Al}$ atoms, one can observe two broadlines centred at 56 and $7 \mathrm{ppm}$ respectively $(\Delta \nu=5000$ and $2500 \mathrm{~Hz})$ in the intensity ratio $5 / 1$. From their chemical shifts we can assign these resonances to tetrahedral and octahedral species respectively. The broadening is due to highly anisotropic aluminium sites and these resonances arise from $\mathrm{Al}$ atoms in oligomers which possess higher electric field gradi- ents at the aluminium centre than in the monomer. Actually, the sharp line progressively disappears with time (Fig. 1c) while the intensity of the broad line centred at $56 \mathrm{ppm}$ increases, indicating that a growth process takes place where $\mathrm{Al}$ atoms are mainly 4-coordinated. At $t / t_{g} \sim 0.96$ (Fig.1d), only the two broad bands are observed and a partial conversion of tetrahedral $\mathrm{Al}$ to octahedral $\mathrm{Al}$ can be noted. Using NMR peak intensities, one can deduce that $62 \%$ of $\mathrm{Al}$ atoms are in tetrahedral sites and $38 \%$ in octahedral sites.

However, it is difficult to determine how much of $\mathrm{Al}$ in the sample is actually observed during the experiment. Since ${ }^{27} \mathrm{Al}$ is a quadrupolar nucleus, small changes in the local environment surrounding the nucleus can produce dramatic changes in linewidth and intensity in the resonance $[8,9]$ due to electric field gradients. Nevertheless, the gelation process clearly leads to important modifications in the ${ }^{27} \mathrm{Al}$ NMR spectra.

These NMR results demonstrate that near the gel point there is only a very weak concentration of silicic condensed species ( $\mathrm{Si}$ atoms with bridging oxygens) and that inorganic polymerization is essentially due to the formation of Al-O-Al linkages.

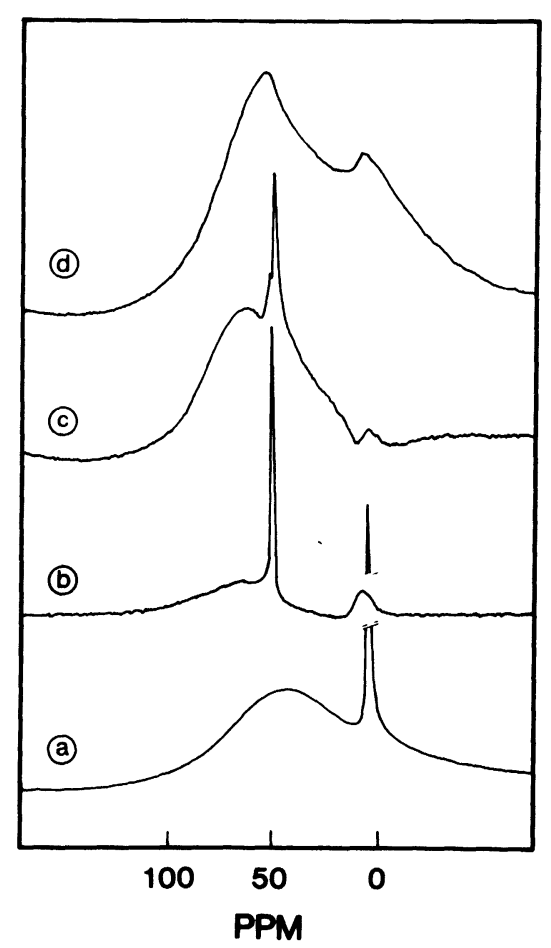

Fig.1.- ${ }^{27} \mathrm{Al}-104 \mathrm{MHz}-\mathrm{NMR}$ spectra. Chemical shifts are relative to $\mathrm{Al}\left(\mathrm{H}_{2} \mathrm{O}\right)_{6}^{3+}$. (a) Pure silicon-aluminium ester. (b) After adding water, $t / t_{g}=0$ (c) $t / t_{g}=0.30$ (d) $t / t_{g}=0.96$

\section{SAXS results}

During the growth process, usual monotonically decreasing SAXS curves are observed (Fig.2). With time, 
we note no change in the high angle part of the SAXS curves while the scattered intensity at low angles increases. Moreover, in agreement with previous observations of Schaefer and Keefer in the silica system [6], we have observed no significant change of the scattering profile to the gel point in spite of a drastic change in mechanical properties at the moment of cross-over from a liquid to a rigid gel with a glassy aspect. This observation suggests that the drastic change of connectivity has no effect on the spatial distribution of atoms, to which SAXS profiles are sensitive.

Scattering in the Guinier region (low angle) depends on the characteristic dimension $R$ of the scattering entities and from the initial decay of the scattered intensity $I(H)$, one can deduce the electronic radius of gyration $R_{\mathrm{g}}$ using the following formula [10] :

$$
\begin{gathered}
I(H) \sim n^{2} N\left(1-H^{2} R_{\mathrm{g}}^{2} / 3\right) \\
\left(R_{\mathrm{g}}=(3 / 5)^{1 / 2} \times R \text { for spherical particles }\right)
\end{gathered}
$$

where $H$ is the scattering wave vector, $N$ is the number of scattering particles and $n$ the number of electrons in each particle.

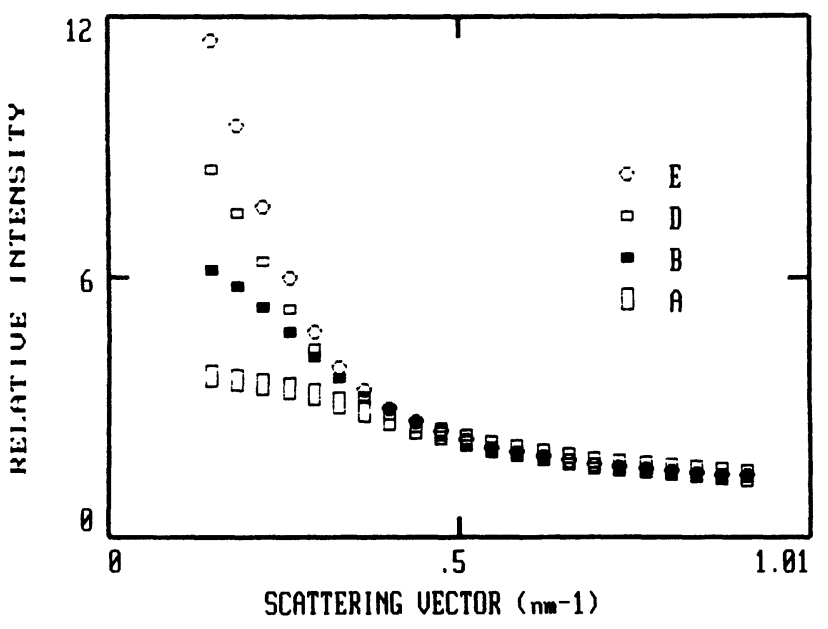

Fig.2.-Time dependence of the scattering profiles during gelation (average over $\left.0.03 t_{g}\right) \mathbf{A}\left(t / t_{g}=0.13\right), \mathrm{B}(t /$ $\left.t_{g}=0.40\right), \mathrm{D}\left(t / t_{g}=0.73\right), \mathrm{E}\left(t / t_{g}=0.93\right)$.

Concerning the silicic ester, first we observe that the Guinier radius does not tend to zero at the initial time (Fig.3). This result is in agreement with the ${ }^{27} \mathrm{Al}$ NMR spectra which show that the initial solution (after adding water) consists of both monomers and some clusters.

Second, as in the silica systems [6], no divergence of $R_{\mathrm{g}}$ is observed at $t_{g}$. As polymerization proceeds, the Guinier radius of the molecules increases (Fig.3), consistent with the growth of the clusters and near $t_{g}$, the value of $R_{\mathrm{g}}$ saturates at $60 \AA$ probably indicating that the clusters begin to overlap and entangle (the same value of $60 \AA$ is measured at $t=1.1 t_{g}$ ). As a consequence, near $t_{g}$, the measured Guinier radius is then JOURNAL DE PHYSIQUE. - T. 48, N 6, JUIN 1987 substantially less than the real radius of gyration. Scattering in the Porod region defined as $H R>>1>>$ $H a$, where $a$ is the monomer size, depends on the geometric structure of the scattering entities [10]. The scattered intensity decays as power law $I(H) \sim H^{-x}$ and theefore, in a Log-Log plot of $I(H)$ versus the scattering factor, the slope is sensitive to the geometry of the scatterers.

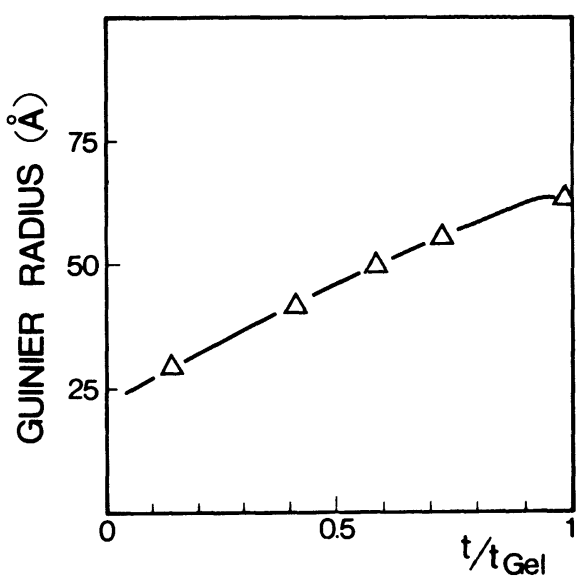

Fig.3.-Time dependence of the Guinier radius measured from the initial curvature of the scattering profiles.

For all the Porot plots (Fig.4), two power law regimes are observed : a slope of $2.60 \pm 0.05$ between 4 and $7 \AA$ and a slope of $1.80 \pm 0.05$ in the $\left(R_{\mathrm{g}} / 2\right)-10 \AA$ length range. Moreover, at about $0.7 t_{g}, S A X S$ was recorded from samples at $1 / 4$ dilution in order to decrease possible interference effects and the same slopes are obtained in the Porod range. There Porod exponents are constant through the gel point indicating that the local structure remains unchanged at gelation.

In fact, the slope of 1.8 is observed, at least near $t_{g}$, over a decade of reciprocal space. Therefore, the exponent, at high length scale (above $10 \AA$ ) can be reasonably equated to the fractal dimension $D$.

The fractal dimension is also found from the $R_{\mathrm{g}}$ dependence of the molecular weight $(M \sim n)$. In the Guinier approximation

$$
I(H=0) \sim n^{2} \times N .
$$

Assuming that $n \times N$ is constant (the total mass in solution remains constant and the particles grow), $I(H=0) \sim$ $n$ and $I(H=0)$ is proportional to the Z-averaged number of atoms in the particles. Therefore, for a massfractal object :

$$
I(H=0) \sim R^{D}
$$

where $D$ is the fractal dimension and $R$ is the average radius of gyration. 


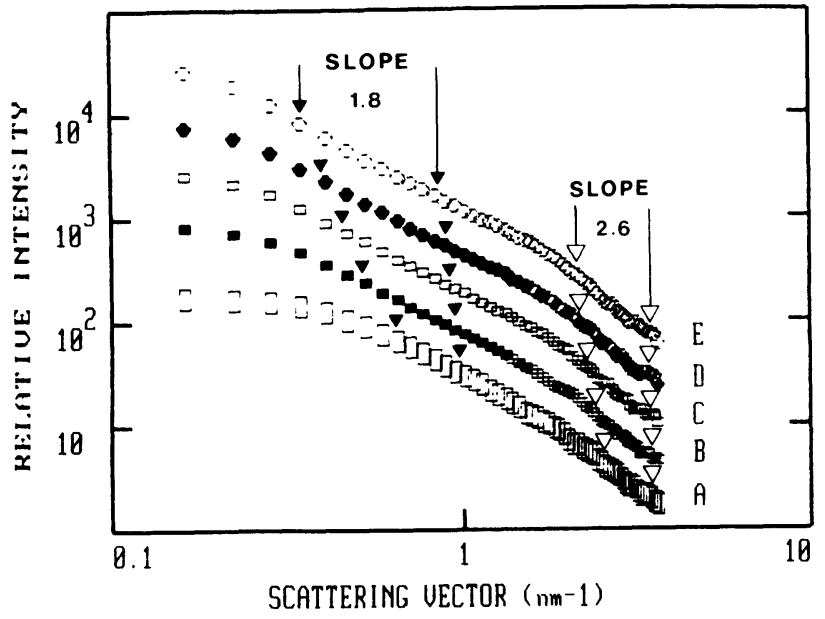

Fig.4.-Time dependence of Porod plots of the scattering curves. The first slope (2.6) is measured between 7 and $4 \AA$ and the second (1.8) between $R_{\mathrm{g}} / 2$ and $10 \AA$ (black arrows). Under the same experimental condictions, the Porod slope is -4 for gold particles. $\mathrm{A}\left(t / t_{g}=0.13\right), \mathrm{B}\left(t / t_{g}=0.40\right), \mathrm{C}\left(t / t_{g}=0.60\right)$, $\mathrm{D}\left(t / t_{g}=0.73\right), \mathrm{E}\left(t / t_{g}=0.93\right)$.

After $1 / 4$ dilution of the sample at $0.7 t_{g}$, no significant change of $R_{\mathrm{g}}$ is noted, displaying that the particles are only slightly overlapped prior to gelation. Consequently, in the range where the Guinier radius equals of gyration, a fractal dimension can be deduced. In fact, a value of $1.75 \pm 0.05$ is found (Fig.5) over a small range of $R_{\mathrm{g}}$, which is very close to the previous experimental fractal dimension.

\section{Discussion}

The first Porod exponent (2.6) is compatible with the existence at very short length scale (5-7 $\AA$ ) of densely crosslinked polymeric particles. In agreement with the value of $R_{g}$ at $t=0$ and the absence of change in the high-angle part of the SAXS curve (no formation of scattering centres during the gelation), we think that these elementary particles exist initially (after adding water) and result from a fast monomer growth process. Considering the NMR results, these polymeric particles are formed of Al-O-Al linkages with fourcoordinated $\mathrm{Al}$ atoms. The chemical growth mechanism taking place from condensation reactions between monomers where only OR groups localized on $\mathrm{Al}$ atoms are fastly hydrolyzed ( $=\mathrm{Al}-\mathrm{OH}+\mathrm{HO}-\mathrm{Al}=\rightarrow=\mathrm{Al}-\mathrm{O}$ $\mathrm{Al}=$ ).

The fractal dimension 1.8 is in agreement with a clustercluster growth mechanism. Concerning the aggregation process, as summarized by Schaefer and Keefer [11], there are two limit models. The first called clustercluster diffusion-limited aggregation has been extensivelly studied by computer simulations $[12,13]$. In this

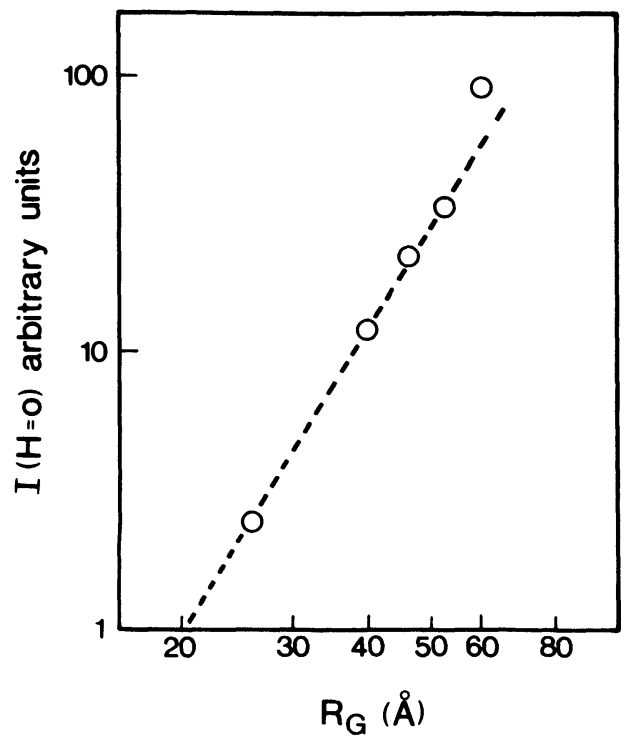

Fig.5.-Log-Log plot of the scattered intensity at $H=0$ (deduced by using the Guinier approximation) versus the radius of gyration.

model, only the clusters of exactly the same number of particles stick after diffusing in a purely random walk fashion in empty space. The result of this process is an open highly ramified object with a fractal dimension of the clusters of 1.78 .

In fact, it is more realistic to assume that the clusters do not stick on every contact. Simulations of diffusionlimited aggregation with very low sticking probability [14] yield a fractal dimension of 2-2.1. This type of aggregation is a reaction-limited process and is now called the reaction-limited cluster aggregation (R.C.L.A.). For instance, polymerizations in the silica [6] and alumina [15] systems lead to a fractal dimension of 2 consistent with the R.C.L.A. model. In contrast, we only found 1.8 for the silicic ester system close to the diffusionlimited aggregation model.

In fact, the gel time (about 15 hours) is probably incompatible with a pure diffusion-limited mechanism which implies a very short aggregation time. Nevertheless, our system seems to exhibit a special behaviour compared to pure silica and alimina systems. Moreover, the same fractal dimension 1.8 has been observed in aluminosiloxane gels which consist of Al-O-Al linkages and unreactive $\mathrm{O}-\mathrm{Si}\left(\mathrm{CH}_{3}\right)_{3}$ groups localized on each $\mathrm{Al}$ atom [1].

This fractal dimension may be due to the large $\mathrm{Si}(\mathrm{OR})_{3}$ or $\mathrm{Si}\left(\mathrm{CH}_{3}\right)_{3}$ unhydrolyzed groups which reduce the degree of branching and probably lead to orientational effects for hydrolysis-condensations reactions. Axelos et al. [16] have observed a dimension 1.4 in an alumina system in which a two-dimensional aggregation seems to take place. However, Schaefer and Keefer [6] claimed that the degree of branching does not influence the fractal dimension in the silica system although the experimental Porod slope varies from 1.9 to $2.1 \mathrm{de}$ - 
pending on the acid or base-catalyzed conditions and on the precursor/water molar ratio.

\section{Conclusion}

This study clearly shows that the kinetics of polymerization (hydrolysis-condensations) is very different in the aluminium and silicon systems. In our experimental conditions the polymerization of the Al-O-Si precursor only implies the formation of Al-O-Al linkages. After adding water and dissolving a precipitate, the sol consists of small elementary clusters (5-8 $\AA$ ) which can be equated to dense polymeric with four-coordinated $\mathrm{Al}$ atoms and which are probably reminiscente of the precipitate.

With time, a cluster-cluster aggregation takes place characterized by a fractal dimension 1.8. According to NMR results, clusters probably stick together with a partial conversion of tetrahedral Al to octahedral Al. This suggests that aggregation is associated not only the nucleophilic reaction between two Al-OH groups but also with a change of the Al coordination.

\section{References}

(1] Pouxviel, J.C., Boilot, J.P., DAuger, A. and HuBER, L., in Better Ceramics Through Chemistry II, edited by C.J. Brinker, D.R. Ulrich and D.E. Clark, (North Holland-Elsevier) 1986, Vol.73 p269.

[2] Engelhardt, G., Atlenburg, W. Hoebbel, D. and Wicker, W., Z. Anorg. Allg. Chem. 428 (1977) 43.

[3] HaRris, R.K. and KNIGHT, C.T.G., J.C.S. Chem. Comm. 421 (1980) 756.

[4] ARtaki, I., BRAdley, M., Zerda, T.W. and JoNAS, J., J. Phys. Chem. 89 (1985) 4399.
[5] Pouxviel, J.C., Bollot, J.P., Beloeil, J.C. and Lallemand, J.Y., Non-Cryst. Solids to be published.

[6] Schaefer, D.W. and Keefer, K.D., Phys. Rev. Lett. 53 (1984) 1383 and Fractals in Physics, edited by L. Pietronero and E. Tosatti (Elsevier Science Publishers B.V.) 1986, p.39.

[7] HARRIS, R.K. and MANN, E.M., NMR and the Periodic Table (Academic Press) 1978.

[8] FenzKe, D. Freude, D., Frohlich, T. and HaEse, J., Chem. Phys. Lett. 111 (1984) 171.

[9] Olson, W.L. and BAUER, L.J., Better Ceramics Through Chemistry II, edited by C.J. Brinker, D.R. Ulrich and D.E. Clark, (North-Holland-Elsevier) 1986, Vol.73, p.187.

[10] GuINIER, A. and Fournet, G., Small-Angle Scattering of $X$-rays (John Wiley and Son, N.Y.) 1985.

(11] SChAEFER, D.W. and KeEFeR, K.D., in Better Ceramics Through Chemistry II, edited by C.J. Brinker, D.R. Ulrich and D.E. Clark, (North-Holland-Elsevier) 1986, Vol.73, p.277.

[12] Meakin, P., Phys. Rev. Lett. 51 (1983) 1119.

[13] Kolb, M., Botet, R. and Jullien, R., Phys. Rev. Lett. 51 (1983) 1123.

[14] Kolb, M., Botet, R. and Jullien, R., Phys. Rev. Lett. 45 (1983) L-211.

[15] Schaefer, D.W., Shelleman, R.A., Keefer, K.D., and MARTIN, J.E., Physica A140 (1986) 105.

[16] Axelos, M., Tchoubar, D., Bottero, J.Y., and Fiessinger, F., J. Physique 46 (1985) 1587. 